\title{
Editorial
}

\section{Changing trends in use of hepatocytes and microsomes for evaluating metabolism studies in drug discovery}

\author{
Abdul Naveed Shaik \\ Guest Editor: ADMET \& DMPK \\ Center for Pharmacometrics and Systems Pharmacology, College of Pharmacy, University of Florida, Orlando, FL \\ Email: naveedshaik@gmail.com; Tel.: +1-217-413-7760.
}

A superfamily of hemoprotein named cytochrome P450 (CYPs) are the proteins which bring about catalysis of many endo biotics as well as xenobiotics. These are the membrane associated proteins located in the endoplasmic reticulum. As these proteins bring about oxidative metabolism of many xenobiotics, they have been since used to in drug discovery and development extensively to study the drug metabolism of new chemical entities (NCEs). In humans, these CYPs are localized in different organs, such as liver, brain, intestine and kidney. In the liver, CYPs are present in the endoplasmic reticulum of hepatocytes. Ultracentrifugation of hepatocytes at a speed of $100,000 \mathrm{Xg}$ will generate microsomes.

Microsomes have been traditionally used in drug discovery to evaluate the metabolic properties of NCEs. They contain both Phase I enzymes such as a CYPs, Flavin Mono Oxygenase (FMO) and Phase II enzymes such as Uridine 5'-diphospho-glucuronosyltransferases (UGTs), which can be regarded as a convenient metabolic tool in drug discovery. There are few limitations of microsomes. For instance, they have to be supplied with co-factors like NADPH, or UDPGA for the metabolic reactions to initiate, and lack the cell membranes to mimic the physiological environment in hepatocytes.

However, in the cell system the drugs never have easy access to the drug metabolizing enzymes. They have to pass through barriers which will have drug transporters facilitating the movement of these drugs across the membrane either concentrating the cell, granting access or effluxing the drug and denying the drugs access to the enzymes. Due to the involvement of these drug transporters, in drug disposition and drug-drug interactions, there has been major interest in the past decade to study the role of these transporters in drug discovery and development. Hepatocytes have a cellular organization containing, the necessary co-factors and membranes transporters, which represent a more complete system to study drug metabolisms. Fresh cryopreserved hepatocytes have been made commercially available in recent years. There has been a change in trend to use hepatocytes in drug discovery and development. Due to the high cost of hepatocytes and the complexity in conducting experiments with hepatocytes, microsomes still remain the tool of choice for early drug discovery studies.

ADMET \& DMPK devoted a special issue on use of microsomes and hepatocytes in drug discovery with the aim to analyze the trend in use of these tools in drug discovery and development. There have been few original articles as well as review articles submitted to this special issue, these articles covered varied areas including, fetal hepatocytes, pharmacogenetics profiling and metabolic activity in stem cell derived 
hepatocytes, microsomes in brain, ADMET properties of natural products and an opinion on use of hepatocytes and microsomes in various stages of drug discovery and development. Clearly microsomes and hepatocytes are playing an important role in supporting the metabolic study in drug discovery. In the near future, we will call for more special issues related to drug metabolisms and drug transporters.

(C2016 by the authors; licensee IAPC, Zagreb, Croatia. This article is an open-access article distributed under the terms and conditions of the Creative Commons Attribution license (http://creativecommons.org/licenses/by/3.0/) (cc)) EY 